\title{
Shape-based Discrimination and Classification of Cortical Surfaces
}

\author{
Peng $\mathrm{Yu}^{1}$, Xiao Han ${ }^{2,}$ Florent Ségonne ${ }^{3}$, Arthur K. Liu ${ }^{4}$, Russell A. Poldrack ${ }^{5}$, Polina Golland ${ }^{3}$, \\ Bruce Fischl $1,3,4,6$ \\ ${ }^{1}$ Health Science and Technology, MIT, Cambridge, MA, 02139, United States; ${ }^{2}$ CMS Inc., St. \\ Louis, MO, 63132; ${ }^{3}$ MIT, AI lab, Cambridge, MA, 02139, United States; ${ }^{4}$ Radiology, MGH, \\ Charlestown, MA, 02129, United States, ${ }^{5}$ Department of Psychology and Brain Research \\ Institute, UCLA, Los Angeles, CA, 90095-1563, United States; ${ }^{6}$ Athinoula A. Martinos Center for \\ Biomedical Imaging, MGH/MIT/HMS, Charlestown, MA, 02129, United States;
}

\begin{abstract}
Advances in medical imaging technique make it possible to study shape variations of neuroanatomical structures in vivo, which has been proved useful in the study of neuropathology and neurodevelopment. In this paper, we propose the use of spherical wavelet transformation to extract shape features, as it can characterize the underlying functions in a local fashion in both space and frequency, in contrast to spherical harmonics that have a noncompact basis set. The extracted shape features can be used to statistically detect and visualize group shape differences from a coarse to fine resolution, and facilitate shape-based classification. A procedure is developed to apply this method to cortical surface models, and promising results are acquired on synthetic and real data.
\end{abstract}

\section{Introduction}

Evidence suggests that morphological changes of neuroanatomical structures may reflect abnormalities in neurodevelopment, or a variety of disorders. The morphological variations can be characterized by the change of volume and shape. Research in this field has been largely focused on the relationship between pathology and volumetric variations of various neuroanatomical structures. Recently, a considerable amount of work has been conducted to measure the changes in the 2D or 3D shape of brain structures, with the goals of more accurate diagnoses, better treatments, and an improved understanding of neuropathology and neurodevelopment.

To accurately study inter-subject shape variations, one would like to find not only an effective shape representation but also a registration method to preserve individual variation while aligning anatomically important structures. Different techniques employed to address these two problems contribute to the merits and disadvantages of various shape analysis methods. One of the earliest methods developed in this field represented shape by points sampled on the boundary of the object being studied. The coordinates of the corresponding points on different subjects were directly used as shape features [1]. Cootes et al. extended this method by applying principle component analysis (PCA) on the point distribution model, which allows for a global scale shape analysis [2]. However, this method relies on the accuracy of the inter-subject registration for group comparison. Subsequently, parametric models were developed to decompose the boundary or surface using Fourier descriptors or spherical harmonics (SPHARM), and to use the decomposition coefficients as shape descriptors [3-5]. A drawback of these models is the inadequacy of spatial localization due to the global support of the basis functions. Another popular method warps a template to individual subjects and studies the deformation field for shape variations [6]. This method has shown promising results, but it is sensitive to the template selection and presents challenges in interpreting and comparing shape differences using the high-dimensional deformation field. The medial axis technique, originally proposed by Pizer et al. and Golland et al. in 3D and 2D respectively, has been applied as a powerful tool on the shape analysis of a variety of subcortical structures $[7,8]$. This technique provides an intra-subject correspondence and allows for the separate studies of the local position and thickness of the object at both coarse and fine levels, due to the merits of its mathematical expression. However, the sensitivity to small perturbations in the boundary is a fundamental problem of this method. 


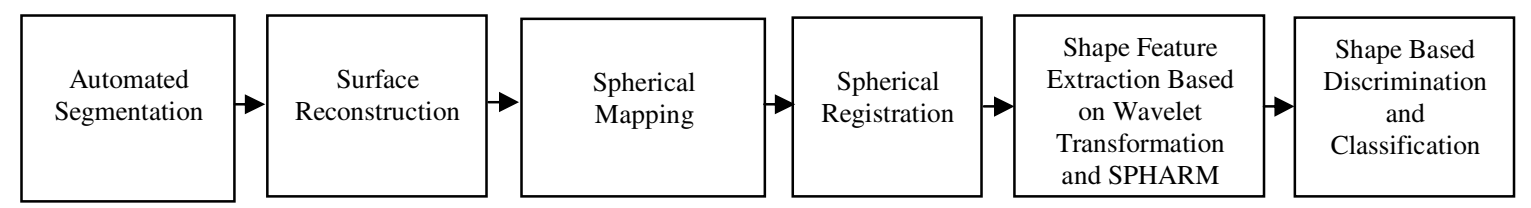

Fig. 1 The automated procedure for conducting shape analysis of neuroanatomical structures

In order to accurately extract shape features and conduct statistical analysis, we developed a procedure to hierarchically study shape variations of cortical surfaces using spherical wavelet transformation, which can characterize the underlying functions in a local fashion in both space and frequency. The entire procedure is introduced in detail in section 2. In section 3 , we demonstrate the use of spherical wavelet transformation in detecting shape variation and compare it with SPHARM. We also present the results of applying this method in cortical shape discrimination and classification, using both synthetic and real neurodevelopment data.

\section{Method}

\subsection{Overall Procedure}

The automated procedure for conducting shape analysis, using spherical wavelet transformation, is shown in Fig. 1. For decomposing a surface using basis functions defined in the spherical coordinates system, such as SPHARM or spherical wavelets, the surface must be mapped onto a parameterized sphere. Tools developed by the FreeSurfer group are used for pre-processing, which includes cortical surface segmentation, cortical surface reconstruction, spherical mapping and registration based on this spherical representation [9, 10]. Spherical wavelet transformation is then applied to the normalized surface and the decomposition coefficients are used as shape features. For group comparison, Hotelling's $\mathrm{T}^{2}$ test is applied to determine the discriminative power of each coefficient. The coefficients with statistic values larger than a prespecified threshold are selected for discrimination analysis and classification. To visualize the shape difference between two groups, we gradually add the differences of the mean values of selected coefficients between Group 1 and Group 2 to the mean wavelet coefficients of Group 1, and inversely transform the resulting new sets of coefficients into surfaces. Visualizing this difference allows us observe the shape variations from a coarse to fine resolution. Finally, leave-one-out classification accuracy is calculated as the percentage of subjects correctly classified by selecting features and training a classifier using the

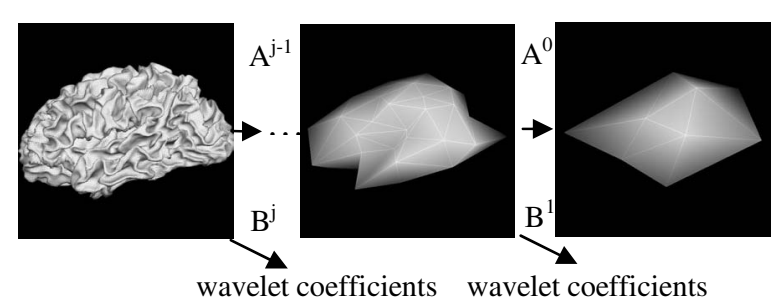

Fig. 2 Wavelet decomposition of cortical surface

remaining subjects. The details of each step are introduced in the following subsections.

\subsection{Spherical Harmonic Descriptors}

As a natural extension of Fourier transformation on the sphere, SPHARM has been demonstrated to be a powerful tool in describing the boundary of objects of spherical topology. In this method, the coordinates $\bar{v}(\theta, \varphi), \theta \in[0, \pi], \phi \in[0,2 \pi)$, of a parameterized surface are expressed as the weighted summation of a set of spherical harmonic basis functions of degree $l$ and order $m$ $Y_{l}^{m}, \quad-l \leq m \leq l$ :

$$
\bar{v}(\theta, \varphi)=\sum_{l=0}^{\infty} \sum_{m=-l}^{l} \bar{c}_{l}^{m} Y_{l}^{m}(\theta, \varphi),
$$

where the coefficients $\bar{c}_{l}^{m}$ contain hierarchical shape information. However, SPHARM coefficients cannot indicate the type and location of shape differences due to the global support property of the basis functions.

\subsection{Spherical Wavelets}

In contrast to the SPHARM coefficients, a wavelet representation of a function consists of a coarse overall approximation and coefficients that influence the function at various details and locations. The classical form of wavelet analysis decomposes signals onto a set of basis functions, called wavelets, in which every wavelet is a scaled and translated copy of a single unique function, called the mother wavelet. However, this shift-invariant theory breaks down when representing data sets defined on bounded surfaces. The newly developed biorthogonal spherical wavelet basis functions are based on recursive subdivision starting with an icosahedron and a lifting scheme [11]. 
Similar to the SPHARM method, the coordinates function $\bar{v}=(x, y, z)^{T}(x, y, z$ are coordinates on the original surface) can be considered a function defined on the sphere and expanded by a set of spherical wavelet functions as:

$$
\bar{v}=\sum_{j, k} \bar{\gamma}_{j, k} \psi_{j, k},
$$

where $\bar{\gamma}_{j, k}$ are the 3-dimensional coefficients at level $j, j=-1,0, \ldots l$ and location $k, k \in M(j)$. A fast wavelet transformation algorithm [11] is employed for efficient and accurate calculation of the coefficients, each of which provides some limited information about both the position and frequency of the decomposed function. Wavelet transformation can capture shape changes with fewer coefficients, as the coefficients in the lowest level provide an overall approximation, and localized morphological variations are captured hierarchically by the higher-level coefficients (Fig.2).

\subsection{Feature Selection}

In the group comparison study, we need to select the coefficients that best separate the groups. In this study we employed Hotelling's $\mathrm{T}^{2}$ test, which is a multivariate analog to student's t test and can be used to test the equivalence of a coefficient vector calculated for two groups. If the value of this statistic is greater than the tabulated distribution for a chosen significance level $\alpha$, then the coefficients are considered to be significantly different between the two groups [12]. In shape analysis, it has been a concern that the large number of features, and therefore the large number of statistical tests involved, increases the chance of incorrect detection. The biorthogonality of the spherical wavelets allows us to select features at each level separately and adjust for multiple comparisons.

\subsection{Classification}

The classification method we employed is the support vector machine (SVM) method, which was originally introduced by Vapnik in 1979. This method has only received extensive attention recently for its application in pattern recognition [13]. Classification using SVM does not require assumptions on the distributions of the input data or a large number of classifier parameters with an increase of data complexity. Therefore, SVM can be applied to classification problems with high-dimensional data and small sample size, as is the case for our data in which we have hundreds of thousands of potential features, and only dozens of subjects.

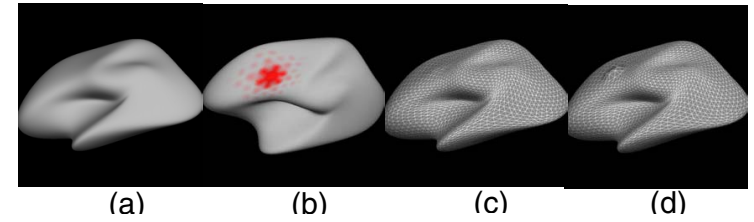

Fig. 3 Comparison of SPHARM and Wavelets

\section{Results}

\subsection{Comparison of Wavelets with SPHARM}

To compare the abilities of SPHARM and spherical wavelets to detect local shape variation, both methods were applied to decompose an inflated cortical surface model with a synthesized shape deformation. The deformed surface was reconstructed using the original surface's coefficients as well as the coefficients calculated from the deformed surface with the most variations, using both SPHARM and spherical wavelets. The coefficient variation is calculated by $\left|\frac{c_{d}-c_{o}}{c_{o}}\right|$, where $c_{d}$ and $c_{o}$ are the corresponding coefficients calculated from the deformed and original surfaces respectively. The reconstruction error is measured by $\frac{1}{N} \sum_{i=1}^{N}\left|\bar{x}_{r}^{i}-\bar{x}_{d}^{i}\right|$, where $\bar{x}_{r}^{i}$ and $\bar{x}_{d}^{i}$ are the coordinates of the $i^{\text {th }}$ vertex on the reconstructed and deformed surfaces, and $N$ is the number of vertices on the surface. Fig. 3(a) is the original surface and 3(b) shows the set of wavelet coefficients with variation values larger than a prespecified threshold are located around the deformation (indicated by red dots). On the other hand, most of the SPHARM coefficients do have variations larger than the threshold. The synthetic bump is barely reconstructed (Fig. 3(c)), with 200 most varied SPHARM coefficients, while this deformation is well reconstructed using the same amount of spherical wavelet coefficients (Fig. 3(d)). A quantitative comparison of the reconstruction error is given in Table 1.

Table 1 Reconstruction error (\%) using varying number of coefficients

\begin{tabular}{|c|c|c|c|}
\hline \# of Coef & 10 & 100 & 200 \\
\hline SPHARM & 96.7 & 79.3 & 67.2 \\
\hline Wavelets & 57.2 & 3.55 & $5 \mathrm{e}-4$ \\
\hline
\end{tabular}

\subsection{Shape Discrimination on Synthetic Data}

The statistical shape analysis procedure is tested on 84 normal brain scans, with half of them having a synthetic $4 \mathrm{~mm}$ by $2 \mathrm{~mm}$ bump around a point selected on the bank of central sulcus. Hotelling's $\mathrm{T}^{2}$ test shows that only coefficients located around the synthetic deformation have a calculated $p$ value lower 


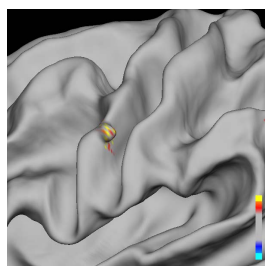

Fig. 4 Statistical map of selected coefficients shown on average deformed surface

fetection rate comparisons correction.

\subsection{Children/Adults Brain Comparison Study}

The brain undergoes dynamic changes through adolescence. However, the specific pattern of development and the relationship of structural change to functional development are still poorly understood. In this experiment, we conduct a pilot study on the developmental differences between children and young adults using our statistical shape analysis method. In particular, shape differences of the gray/white boundary between a set of children (11 controls, 4 female, right-handed, average age 11.83) and young adults (12 controls, 7 female, right-handed, average age 22.17) are analyzed and visualized (left and right hemispheres studied separately). Contrary to the fine-scale deformation we synthesized in section 3.2 , most significant variations are observed at a low spatial-frequency level in this study. Specifically, there are distinct variations in the frontal and temporal lobes in the left hemisphere, and dispersed variations in the parietal lobe in right hemisphere (Fig. 5). Furthermore, leave-one-out classification using low frequency coefficients selected with threshold $=0.01$, gives an accuracy of $73.91 \%$ in the left hemisphere, and $60.87 \%$ in the right hemisphere. These results provide further characterization of the anatomic changes that occur in the normally developing brain.

\section{Conclusion}

Spherical wavelet transformation was demonstrated to be able to accurately and efficiently detect the locations and spatial scales of shape variations. The use of wavelet coefficients in statistical shape analysis has provided a novel way to detect and visualize group differences. Currently improvements are made by using better feature selection and multiple

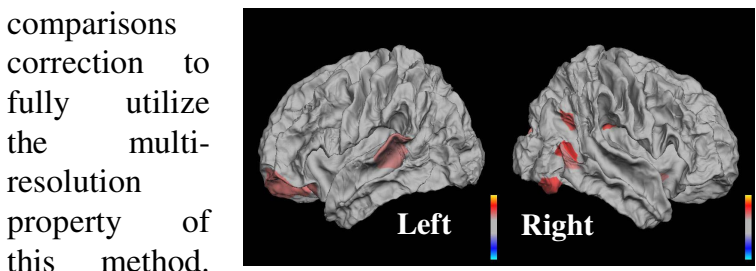

this method.

Future work includes employing advanced statistical tools and extending this method to
Fig. 5 Statistical maps of shape differences between children and young adults of left and right white/grey boundary with brighter color indicating larger significance. other neuroanatomical structures.

\section{References}

[1] F. L. Bookstein, "Shape and the Information in Medical Images: A Decade of the Morphometric Synthesis," Computer Vision and Image Understanding, vol. 66, pp. 97-118, 1997.

[2] T. Cootes, C. Taylor, D. Cooper, and J. Graham, "Active shape models - their training and application," Computer Vision and Image Understanding, vol. 61, pp. 38-59, 1995.

[3] A. Kelemen, G. Székely, and G. Gerig, "Elastic model-based segmentation of 3d neuroradiological data sets," IEEE Transactions on Medical Imaging, vol. 18, pp. 828-839, 1999.

[4] C. Brechbühler, G. Gerig, and O. Kubler, "Parameterization of Closed Surfaces for 3-D Shape Description," Computer Vision and Image Understanding, vol. 61, pp. 154-170, 1995.

[5] L. Shen, Ford J., F. Makedon, and A. Saykin, "hippocampal shape analysis surface-based representation and classification," SPIE Medical Imaging, 2003.

[6] J. G. Csernansky, L. Wang, D. Rastogi-Cruz, J. Posener, N. Brickel, G. Heydebrand, J. Philip Miller, and M. I. Miller, "Hippocampal Deformities in Schizophrenia Characterized by high Dimensional Brain mapping," The American Journal of Psychiatry, vol. 159, pp. 1-7, 2002.

[7] S. M. Peter, G. Gerig, C. S. Joshi, and R. S. Aylward, "Multiscale medial shape-based analysis of image objects," presented at Proceedings of the IEEE, 2003.

[8] P. Golland, W. Grimson, and R. Kikinis, "Statistical shape analysis using fixed topology skeletons: Corpus callosum study," Information Processing in Medical Imaging, pp. 382-388, 1999.

[9] A. M. Dale, B. Fischl, and M. I. Sereno, "Cortical surface-based analysis. I. Segmentation and surface reconstruction," Neuroimage, vol. 9, pp. 179-94., 1999.

[10] B. Fischl, M. I. Sereno, and A. M. Dale, "Cortical surface-based analysis. II: Inflation, flattening, and a surface-based coordinate system," Neuroimage, vol. 9, pp. 195-207., 1999.

[11] P. Schröder, W. Sweldens, "Spherical wavelets: Efficiently representing functions on a sphere," presented at Computer Graphics Proceedings (SIGGRAPH 95), 1995.

[12] D. F. Morrison, Multivariate Statistical Methods. McGraw-Hill. pp.141-145, 1990.

[13] V. Vapnik.. The Nature of Statistical Learning Theory. SpringerVerlag, New York, 1995. 